\title{
Matter-Driven Change of Spacetime Topology
}

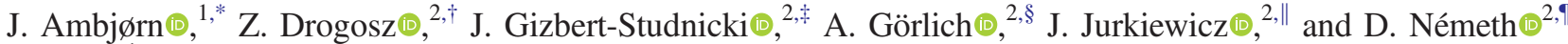 \\ ${ }^{1}$ The Niels Bohr Institute, Copenhagen University, Blegdamsvej 17, DK-2100 Copenhagen Ø, Denmark \\ ${ }^{2}$ Institute of Theoretical Physics, Jagiellonian University, Łojasiewicza 11, Kraków, PL 30-348, Poland
}

(Received 13 March 2021; revised 26 June 2021; accepted 13 September 2021; published 12 October 2021)

\begin{abstract}
Using Monte Carlo computer simulations, we study the impact of matter fields on the geometry of a typical quantum universe in the causal dynamical triangulations (CDT) model of lattice quantum gravity. The quantum universe has the size of a few Planck lengths and the spatial topology of a three-torus. The matter fields are multicomponent scalar fields taking values in a torus with circumference $\delta$ in each spatial direction, which acts as a new parameter in the CDT model. Changing $\delta$, we observe a phase transition caused by the scalar field. This discovery may have important consequences for quantum universes with nontrivial topology, since the phase transition can change the topology to a simply connected one.
\end{abstract}

DOI: 10.1103/PhysRevLett.127.161301

Introduction.-The problem of merging general relativity and quantum mechanics in a theory of quantum gravity has been approached from many directions (string theory [1], loop quantum gravity [2], and the so-called asymptotic safety program using conventional quantum field theory [3], to mention some of the approaches), but no completely satisfactory formulation has yet been found. Difficulties occur already for the pure gravity case, but an additional complication comes from the fact that any realistic theory of quantum gravity should also include coupling to matter fields. The question arises: what type of matter can be included in a particular approach and what impact does it have on the underlying (quantum) geometric degrees of freedom? In this Letter we argue that the impact of matter can be quite dramatic even leading to a change of the topology of the Universe.

Causal dynamical triangulations.-Our attempt to examine the above-mentioned question is via a nonperturbative lattice approach to quantum gravity with the name causal dynamical triangulations (CDT) - see [4] for its detailed formulation and [5] for a recent review. It is an approach which lies within the asymptotic safety program and is only using ordinary quantum field theory concepts. In CDT, the (formal) path integral of quantum gravity is lattice regularized as a sum over four-dimensional simplicial complexes, called triangulations, which encode geometric degrees of freedom; crucially, they are assumed to be endowed with a causal structure of a globally hyperbolic manifold (i.e., spacetime is foliated into spatial

Published by the American Physical Society under the terms of the Creative Commons Attribution 4.0 International license. Further distribution of this work must maintain attribution to the author(s) and the published article's title, journal citation, and DOI. Funded by SCOAP. hypersurfaces of fixed and identical topology), which allows a well-defined Wick rotation of the time coordinate. Thus,

$$
\begin{aligned}
\mathcal{Z}_{Q G} & =\int \mathcal{D}_{\mathcal{M}_{H}}[g] \int \mathcal{D} \phi e^{i S_{\mathrm{EH}}[g]+i S_{M}[\phi, g]} \rightarrow \\
& \rightarrow \sum_{T \in \mathcal{T}} \int \mathcal{D} \phi e^{-S_{R}[T]-S_{M}^{\mathrm{CDT}}[\phi, T]}=\mathcal{Z}_{\mathrm{CDT}},
\end{aligned}
$$

where $\mathcal{M}_{H}$ is a globally hyperbolic Lorentzian manifold, $\mathcal{D}_{\mathcal{M}_{H}}[g]$ denotes the integration over geometries, i.e., equivalence classes of metrics $[g]$ on $\mathcal{M}_{H}$ with respect to diffeomorphisms, and $\mathcal{T}$ is a suitable set of Wick-rotated (now Euclidean) triangulations. The action $S_{R}[T]$ for a triangulation $T \in \mathcal{T}$ is the Einstein-Hilbert action $S_{\mathrm{EH}}$ computed using Regge's method of describing piecewise-linear geometries [6] and containing the bare couplings related to the cosmological and Newton constants. The second term of the action $S_{M}^{\mathrm{CDT}}[\phi, T]$ is the discrete version of the continuous action $S_{M}$ for matter field(s) $\phi$.

Quantum matter fields in CDT.-The simplest quantum matter that can be added to the quantum geometry of CDT is a $d$-component massless scalar field $\phi$. In general, one can assume that the field $\phi$ has a nontrivial target space, i.e., it is a map $\mathcal{M}_{H}\left(g_{\mu \nu}\right) \rightarrow \mathcal{N}\left(h_{\alpha \beta}\right)$ between an arbitrary manifold $\mathcal{M}_{H}$ [from the path integral (1)] with a metric $g_{\mu \nu}$ and a target space $\mathcal{N}$ with some fixed metric $h_{\alpha \beta}$ and fixed topology. The continuous (Euclidean) action $S_{M}$ takes the form

$$
\begin{aligned}
S_{M}[\phi, g]= & \frac{1}{2} \int d^{4} x \sqrt{g(x)} g^{\mu \nu}(x) h_{\rho \sigma}\left[\phi^{\gamma}(x)\right] \\
& \times \partial_{\mu} \phi^{\rho}(x) \partial_{\nu} \phi^{\sigma}(x) .
\end{aligned}
$$


Here, we choose the target space $\mathcal{N}$ of the scalar field to have either Euclidean $\mathbb{R}^{d}$ or toroidal $\left(S^{1}\right)^{d}$ topology, and we fix the flat metric $h_{\rho \sigma}=\delta_{\rho \sigma}$ on $\mathcal{N}$. Consequently, the action (2) reads

$$
S_{M}[\phi, g]=\frac{1}{2} \sum_{\sigma=1}^{d} \int d^{4} x \sqrt{g(x)} \partial^{\nu} \phi^{\sigma}(x) \partial_{\nu} \phi^{\sigma}(x),
$$

and the various components decouple for different $\sigma$ because the target space metric is diagonal. For a particular sample geometry [g], quantum fluctuations of $\phi^{\sigma}$ will occur around a semiclassical solution $\bar{\phi}^{\sigma}$ satisfying the Laplace equation

$$
\Delta_{x} \bar{\phi}^{\sigma}(x)=0, \quad \Delta_{x}=\frac{1}{\sqrt{g(x)}} \partial_{\mu} \sqrt{g(x)} g^{\mu \nu}(x) \partial_{\nu} .
$$

Let us now start with a simple case where the target space of the scalar field is $\mathcal{N}=\mathbb{R}^{d}$. In CDT we consider the scalar field as located at the centers of equilateral simplexes, and thus the discrete counterpart of the action (3) takes a very simple form

$S_{M}^{\mathrm{CDT}}[\phi, T]=\frac{1}{2} \sum_{\sigma=1}^{d} \sum_{i \leftrightarrow j}\left(\phi_{i}^{\sigma}-\phi_{j}^{\sigma}\right)^{2}=\sum_{\sigma, i, j} \phi_{i}^{\sigma} \mathbf{L}_{i j}(T) \phi_{j}^{\sigma}$.

The sum $\sum_{i \leftrightarrow j}$ is over the five pairs of neighboring foursimplexes of each simplex $i$ in the triangulation $T$ of the manifold $\mathcal{M}_{H}\left(g_{\mu \nu}\right)$ and $\mathbf{L}=51-\mathbf{A}$ is the Laplacian matrix, where $\mathbf{A}_{i j}$ is the adjacency matrix with entries of value 1 if simplexes $i$ and $j$ are neighbors and 0 otherwise. The discrete version of the Laplace equation (4) for each component of the classical scalar field is then

$$
\mathbf{L} \bar{\phi}^{\sigma}=0,
$$

which is solved by $\bar{\phi}=$ const (the Laplacian zero mode) for any compact simplicial manifold $T$. After the decomposition of the field

$$
\phi^{\sigma}=\bar{\phi}^{\sigma}+\xi^{\sigma}
$$

into the classical part $\bar{\phi}^{\sigma}$ and the quantum part $\xi^{\sigma}$ and an application of (6), the contribution from the classical field vanishes, leaving

$S_{M}^{\mathrm{CDT}}[\phi, T]=\frac{1}{2} \sum_{\sigma=1}^{d} \sum_{i \leftrightarrow j}\left(\xi_{i}^{\sigma}-\xi_{j}^{\sigma}\right)^{2}=\sum_{\sigma, i, j} \xi_{i}^{\sigma} \mathbf{L}_{i j}(T) \xi_{j}^{\sigma}$.

The Gaussian form of the matter action (8) means that, in principle, the field can be integrated out, contributing to the geometric action $S_{R}[T] \rightarrow S_{R}[T]+S_{M}^{\text {eff }}[T]$ with a term

$$
S_{M}^{\mathrm{eff}}[T]=\frac{d}{2} \log \operatorname{det}\left[\mathbf{L}^{\prime}(T)\right],
$$

where $\mathbf{L}^{\prime}(T)$ is the Laplacian matrix $\mathbf{L}(\mathbf{T})$ in the subspace orthogonal to the constant zero mode of $\mathbf{L}$. The dependence of Eq. (9) on the geometry rests in the dependence of $\mathbf{L}^{\prime}(T)$ on the adjacency matrix $\mathbf{A}$ defined for a given triangulation $T$. Using numerical Monte Carlo simulations we checked that the dependence of the determinant $S_{M}^{\text {eff }}[T]$ on $T$ is weak and, in practice, we can treat it as a constant.

Quantum scalar fields with values on $\left(S^{1}\right)^{d}$.-The new aspect studied here is based on two major generalizations of the CDT model: (1) We choose the spatial topology of the time foliation leaves to be $\left(S^{1}\right)^{3}$, and for technical reasons we assume the time boundary conditions to be periodic as well. Thus, each triangulation has the toroidal topology $\left(S^{1}\right)^{4}$ and can equivalently be represented as an elementary cell periodically repeated in four dimensions. There is a lot of freedom in the selection of the elementary cell; one way to determine it is to choose four independent noncontractible three-dimensional boundaries delimiting it. The boundaries are connected sets of three-dimensional faces, each shared by two four-simplexes located in different copies of the elementary cell. We assume each boundary to be oriented, and we encode the information about the position of the four boundaries (labeled by $\sigma=1,2,3,4$ ) in a triangulation $T$ within four matrices $\mathbf{B}^{\sigma}$, whose elements are

$\mathbf{B}_{i j}^{\sigma}= \begin{cases} \pm 1 & \begin{array}{l}\text { if the face shared by simplexes } i \text { and } j \\ \text { exists and belongs to the boundary }\end{array} \\ 0 & \text { otherwise. }\end{cases}$

The number of directed boundary faces of a simplex $i$ is given by $b_{i}^{\sigma}=\sum_{j} \mathbf{B}_{i j}^{\sigma}$, and the boundary three-volume is $V^{\sigma}=\frac{1}{2} \sum_{i j}\left(\mathbf{B}_{i j}^{\sigma}\right)^{2}$. Despite being fictitious constructs having no impact on the physics, the boundaries can be used to define a coordinate system, as described in $[7,8]$.

(2) The $d$-component scalar field $\phi$ is assumed to take values on a symmetric torus $\mathcal{N}=\left(S^{1}\right)^{d}$ with circumference $\delta$ in each direction. We require that each component of the field $\phi^{\sigma} \in S^{1}$ winds around the circle once as we go around any noncontractible loop in $T$ that crosses a boundary in direction $\sigma$. This requirement completely changes the dynamics of the interaction between geometry and the scalar field. For the scalar field taking values in $\mathbb{R}^{d}$, the classical solution is constant and does not contribute to the matter action, which depends therefore only on quantum fluctuations. For $\phi^{\sigma} \in S^{1}$, however, the constant solution is not allowed, since it has winding number zero; as we will show below, the new nontrivial classical solution does contribute to the effective matter action. The winding condition can technically be obtained by considering a field 
$\phi^{\sigma} \in \mathbb{R}$ that jumps by $\delta \times \mathbf{B}_{i j}^{\sigma}$ on any face shared by simplexes $i$ and $j$ and identifying

$$
\phi_{i}^{\sigma} \equiv \phi_{i}^{\sigma}+n \times \delta, \quad n \in \mathbb{Z} .
$$

The (discrete) matter action becomes

$$
S_{M}^{\mathrm{CDT}}[\phi, T]=\frac{1}{2} \sum_{\sigma=1}^{d} \sum_{i \leftrightarrow j}\left(\phi_{i}^{\sigma}-\phi_{j}^{\sigma}-\delta \times \mathbf{B}_{i j}^{\sigma}\right)^{2}
$$

leading to the following equation for the classical field $\bar{\phi}^{\sigma}$ :

$$
\mathbf{L} \bar{\phi}^{\sigma}=\delta \times b^{\sigma},
$$

which now acquires a boundary term and thus admits nontrivial solutions for $\bar{\phi}^{\sigma}$. Note that the action (12) is invariant under a local shift of the $\sigma$-boundary with a simultaneous change of the scalar field value $\phi_{i}^{\sigma} \rightarrow \phi_{i}^{\sigma} \pm \delta$. Thus the choice of a specific boundary does not influence the path integral (1) in any way. Decomposition (7) of the field into the classical and the quantum part yields

$$
S_{M}^{\mathrm{CDT}}[\phi, T]=\sum_{\sigma, i, j} \xi_{i}^{\sigma} \mathbf{L}_{i j}(T) \xi_{j}^{\sigma}+S_{M}^{\mathrm{CDT}}[\bar{\phi}, T] .
$$

Since $\phi^{\sigma}$ and $\bar{\phi}^{\sigma}$ have winding number one, the fluctuation field $\xi^{\sigma}$ is a scalar field with winding number zero, i.e., an ordinary scalar field taking values in $\mathbb{R}$. The action (14) is then again Gaussian and can be integrated out, now leading to $S_{R}[T] \rightarrow S_{R}[T]+S_{M}^{\mathrm{eff}}[T]+S_{M}^{\mathrm{CDT}}[\bar{\phi}, T]$, where the determinant $S_{M}^{\text {eff }}[T]$ is the same as in Eq. (9). Thus the only difference between the impact of the scalar field in $\left(S^{1}\right)^{d}$ and that of the ordinary field in $\mathbb{R}^{d}$ is the dependence of the effective matter action on the nontrivial classical solution $\bar{\phi}$; note that the size of the jump $\delta$ fixes the scale of the classical field. For $\delta=0$ one recovers the $\mathbb{R}^{d}$ case where $S_{M}^{\mathrm{CDT}}[\bar{\phi}, T]$ is zero (the absolute minimum), as $\bar{\phi}=$ const for any quantum geometry $T$. For $\delta>0$ the constant solution is not allowed, and the action $S_{M}^{\mathrm{CDT}}[\bar{\phi}, T]$ depends on the specific geometry $T$. By adjusting the geometry in a rather drastic way, one is still able to reduce the matter action almost to zero. This is illustrated in Fig. 1 in the simple case of a two-dimensional torus with a onedimensional field $\phi$ changing in the vertical direction, but the argument is clearly valid in higher dimensions, and in fact it only depends on one direction being periodic. The topology in the "transverse" directions can be anything. On the left plot we have a torus with volume $V$ and vertical length $L_{V}$, which is pinched to a cylinder of circumference $\varepsilon$ and length $L$. The total matter action of the field configuration alluded to on the plot is

$$
S_{M}^{\mathrm{CDT}}\left[\phi, T_{L}\right]=\left(\frac{\delta}{L}\right)^{2} L \varepsilon=\delta^{2} \frac{\varepsilon}{L},
$$

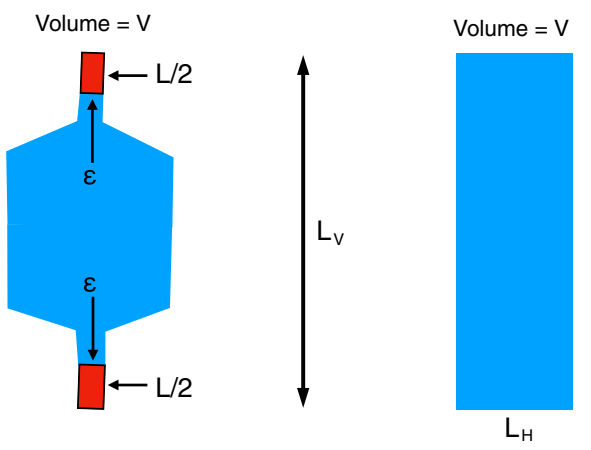

FIG. 1. Left: a torus (opposite sides identified) with a pinch. The region in red is the region where $\phi$ changes from 0 to $\delta$. In the blue part it stays constant. $\phi$ is constant in the horizontal direction. Right: a torus where $\phi$ is constant in the horizontal direction and uniformly increases from 0 to $\delta$ from bottom to top.

and the minimal action for a classical field configuration $S_{M}^{\mathrm{CDT}}\left[\bar{\phi}, T_{L}\right]$ for this geometry is even lower. This can clearly be made arbitrarily small when $\varepsilon \rightarrow 0$, and this is even more true in higher dimensions. On the right plot we also have a torus with volume $V$ and vertical length $L_{V}$. For this geometry, the action is minimal for a field changing uniformly from 0 to $\delta$, when we move from bottom to top, thus obtaining an action

$S_{M}^{\mathrm{CDT}}\left[\bar{\phi}, T_{R}\right]=\left(\frac{\delta}{L_{V}}\right)^{2} L_{V} L_{H}=\delta^{2} \frac{V}{L_{V}^{2}}, \quad V=L_{H} L_{V}$,

which is bounded from below when $V$ and $L_{V}$ are fixed. Let us discuss the consequence of this in the full quantum theory defined by the path integral (1). The classical action $S_{M}^{\mathrm{CDT}}[\bar{\phi}, T]$ depends in a crucial way on the triangulation $T$. The triangulations that are pinched as shown in Fig. 1 will have the smallest matter action, but the geometric EinsteinHilbert part of the action will be larger for such pinched configurations than for "regular" triangulations. A simple minisuperspace model, like the Hartle-Hawking model [9], suggests that for small jumps $\delta$ the geometric part of the action dominates and the generic configurations in the path integral are quite similar to the ones which dominate when no matter field with a jump is present. However, for large $\delta$ the total action will be the lowest for pinched configurations and the system will instead fluctuate around pinched configurations. Thus, the system might undergo a phase transition as a function of the jump magnitude $\delta$.

Results for scalar fields winding around spatial directions.-Below, using numerical Monte Carlo simulations, we study a CDT model with a $d=3$ component massless scalar field taking values in a symmetric torus $\mathcal{N}=\left(S^{1}\right)^{3}$ with circumference $\delta$, i.e., $\phi^{\sigma}$ jumps by $\pm \delta$ when crossing a three-dimensional boundary orthogonal to one of three independent noncontractible loops winding around the toroidal spatial direction $\sigma=x, y, z$ in a 


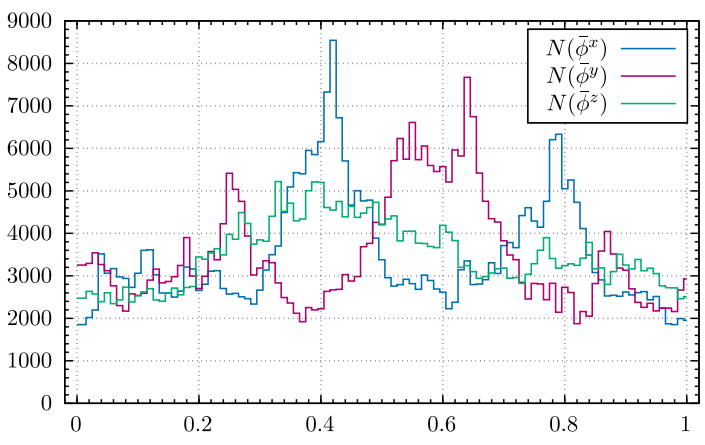

FIG. 2. The projection of four-volume, as defined by (17), on one spatial direction $(x, y$, or $z$ ) for a typical CDT configuration with small jump magnitude ( $\delta=0.1)$. The horizontal axis is $\bar{\phi} / \delta$.

triangulation $T$. This type of matter system was earlier introduced to define a semiclassical coordinate system for a given CDT triangulation [8]. Now we want to make the scalar field a dynamical (quantum) object and let it evolve together with the geometry. Then, for a given geometric configuration $T$, one can simply compute the expectation value of the field $\langle\phi\rangle=\bar{\phi}$ by solving the Laplace equation (13) and use it as spatial coordinates. The analyzed systems were all in the same point in the CDT parameter space inside the so-called semiclassical (or de Sitter) phase [10-12], and the only variable parameter was the jump magnitude $\delta$. For each analyzed value of $\delta$ we pick a generic quantum geometry (a triangulation $T$ ) appearing in the path integral (1), and we use the methodology introduced in [8] to assign a unique set of spatial coordinates for each simplex $i$ defined by the classical solution of the scalar field $\left(\bar{\phi}_{i}^{x}, \bar{\phi}_{i}^{y}, \bar{\phi}_{i}^{z}\right)$ computed for that geometric

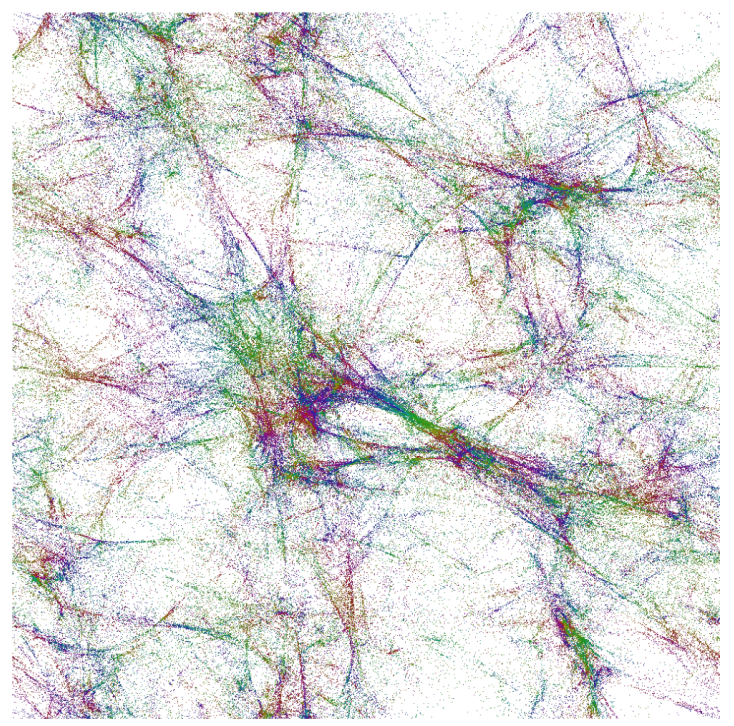

FIG. 3. The projection of four-volume, as defined by (17), on the $x y$ plane for a typical CDT configuration with small jump magnitude $(\delta=0.1)$. Different colors correspond to different times $t$ of the original (lattice) time foliation.

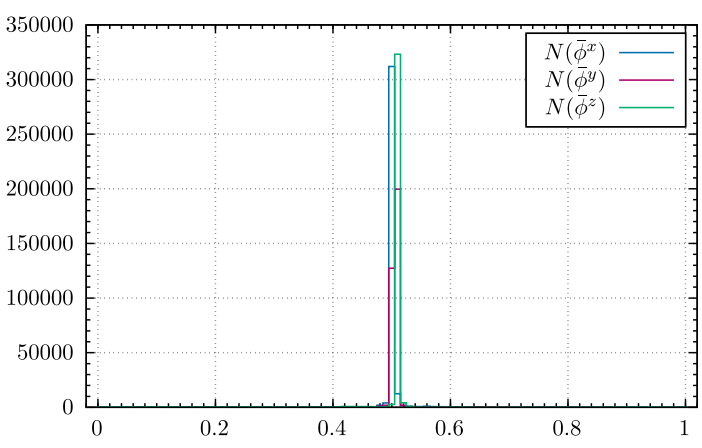

FIG. 4. The projection of four-volume, as defined by (17), on one spatial direction $(x, y$, or $z)$ for a typical CDT configuration with large jump magnitude $(\delta=1.0)$. The horizontal axis is $\bar{\phi} / \delta$.

configuration. Note that in CDT one has the time coordinate "for free" as the classical solution of the field $\bar{\phi}_{i}^{t}$ can be computed using the imposed proper-time foliation. In order to visualize changes in a typical quantum geometry triggered by the increasing jump magnitude, we measure the four-volume density distribution $N(\bar{\phi})$, i.e., the number of simplexes contained in hypercubic blocks with sizes $\left(\Delta \bar{\phi}_{i}^{x}, \Delta \bar{\phi}_{i}^{y}, \Delta \bar{\phi}_{i}^{z}, \Delta \bar{\phi}_{i}^{t}\right)$, which is equivalent to measuring the integrated $\sqrt{g(\bar{\phi})}$ :

$$
\Delta N(\bar{\phi})=\sqrt{g(\bar{\phi})} \prod_{\sigma} \Delta \bar{\phi}^{\sigma}=N(\bar{\phi}) \prod_{\sigma} \Delta \bar{\phi}^{\sigma} .
$$

In Figs. 2 and 4 we plot projections of the volume density distribution $N(\bar{\phi})$ in a typical toroidal CDT configuration on one spatial direction $(x, y$, or $z)$, and in Figs. 3 and 5 the projections on two-dimensional ( $x-y$ directions) parameter

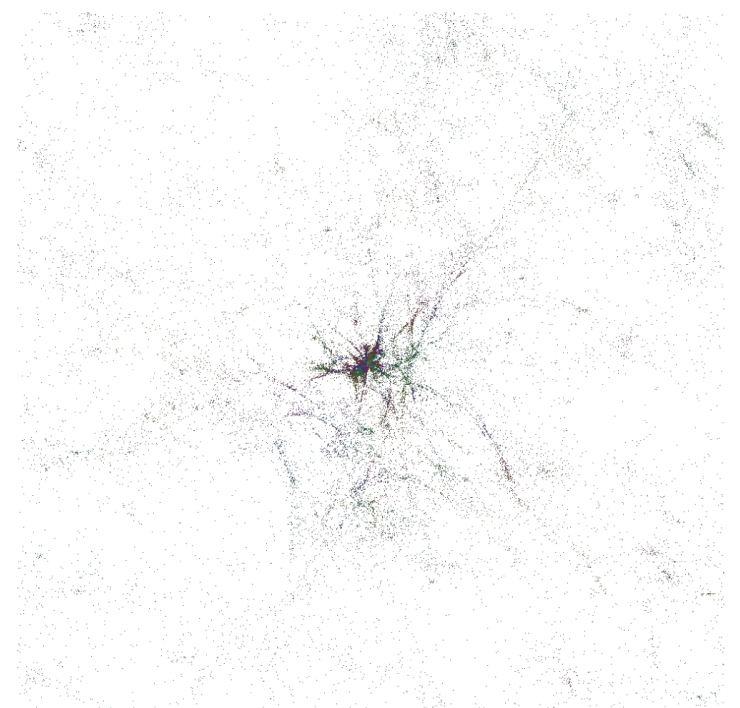

FIG. 5. The projection of four-volume, as defined by (17), on the $x y$ plane for a typical CDT configuration with large jump magnitude $(\delta=1.0)$. Different colors correspond to different times $t$ of the original (lattice) time foliation. 
subspace, integrating over the remaining directions. Figures 2 and 3 are for a small $(\delta=0.1)$, while Figs. 4 and 5 for a relatively large $(\delta=1.0)$ jump magnitude, respectively. One clearly observes that the change of the jump magnitude causes a substantial change in a typical CDT geometry. For a small jump $(\delta=0.1)$ one observes a geometry which resembles the pure gravity case, see [8]. For a large jump $(\delta=1.0)$, in line with expectations, one observes that the geometry is "pinched" in all spatial directions (which manifests itself as the small-volume region in Fig. 4 and the low-density region in Fig. 5).

Discussion.-We have shown that if spacetime is globally hyperbolic and has the toroidal spatial topology, i.e., has three nonequivalent noncontractible loops in the spatial directions, then the three-component scalar field with matching topological boundary conditions imposed can have a dramatic effect on the geometries that dominate the CDT path integral. If the spatial topology is simply connected, this effect is absent. This new kind of coupling between the topology of the matter fields and the topology of spacetime is likely to result in a phase transition for sufficiently strong coupling (sufficiently large $\delta$ in our model), a transition where the path integral will be dominated by spatial geometries with pinched regions fluctuating close to zero sizes (but still connected due to topological restrictions imposed by our model). This is schematically shown in Fig. 1 and for actual configurations in the path integral in Figs. 4 and 5, using as coordinates in the noncontractible "directions" the classical scalar fields with nontrivial boundary conditions in these directions, and what is also visible using other coordinate systems, e.g., the ones introduced in [7]. Extrapolating this result to a large volume limit we get a picture with a small toroidal part of cutoff size and the dominating geometry with an (almost) spherical topology. Concluding, the effect of scalar fields can be much more drastic than previously appreciated, with possible implications for cosmological model building and even in other areas of physics related to phase transitions of topological nature.

Z. D. acknowledges support from the National Science Centre, Poland, Grant No. 2019/32/T/ST2/00390. J. G.-S. acknowledges support of Grant No. 2016/23/ST2/00289 from the National Science Centre Poland. A. G. acknowledges support by the National Science Centre, Poland, under Grant No. 2015/17/D/ST2/03479. J. J. acknowledges support from the National Science Centre, Poland, Grant No. 2019/33/B/ST2/00589. D. N. acknowledges support from National Science Centre, Poland with Grant No. 2019/32/T/ST2/00389.

*ambjorn@nbi.dk.

†bigniew.drogosz@doctoral.uj.edu.pl

*jakub.gizbert-studnicki@uj.edu.pl

§andrzej.goerlich@uj.edu.pl

"jerzy.jurkiewicz@uj.edu.pl

nemeth.daniel.1992@gmail.com

[1] J. Polchinski, String Theory (Cambridge University Press, Cambridge, England, 1998), Vols. 1 and 2.

[2] T. Thiemann, Modern Canonical Quantum General Relativity (Cambridge University Press, Cambridge, England, 2010).

[3] M. Reuter and F. Saueressig, Quantum Gravity and the Functional Renormalization Group: The Road Towards Asymptotic Safety (Cambridge University Press, Cambridge, England, 2019).

[4] J. Ambjørn, A. Görlich, J. Jurkiewicz, and R. Loll, Phys. Rep. 519, 127 (2012).

[5] R. Loll, Classical Quantum Gravity 37, 013002 (2020).

[6] T. Regge, Nuovo Cimento 19, 558 (1961).

[7] J. Ambjørn, Z. Drogosz, J. Gizbert-Studnicki, A. Görlich, and J. Jurkiewicz, Nucl. Phys. 943, 114626 (2019); J. Ambjørn, Z. Drogosz, A. Görlich, and J. Jurkiewicz, Phys. Rev. D 103, 086022 (2021).

[8] J. Ambjørn, Z. Drogosz, J. Gizbert-Studnicki, A. Görlich, J. Jurkiewicz, and D. Németh, Eur. Phys. J. C 81, 708 (2021).

[9] J. B. Hartle and S. W. Hawking, Phys. Rev. D 28, 2960 (1983).

[10] J. Ambjø rn, J. Jurkiewicz, and R. Loll, Phys. Rev. Lett. 93, 131301 (2004); Phys. Lett. B 607, 205 (2005); J. Ambjørn, A. Görlich, J. Jurkiewicz, R. Loll, J. Gizbert-Studnicki, and T. Trzesniewski, Nucl. Phys. B849, 144 (2011).

[11] J. Ambjørn, J. Jurkiewicz, and R. Loll, Phys. Rev. D 72, 064014 (2005); J. Ambjørn, A. Görlich, J. Jurkiewicz, and R. Loll, Phys. Rev. D 78, 063544 (2008); Phys. Rev. Lett. 100, 091304 (2008).

[12] J. Ambjørn, Z. Drogosz, J. Gizbert-Studnicki, A. Görlich, J. Jurkiewicz, and D. Németh, Phys. Rev. D 94, 044010 (2016); J. Ambjørn, J. Gizbert-Studnicki, A. Görlich, K. Grosvenor, and J. Jurkiewicz, Nucl. Phys. B922, 226 (2017). 